\title{
Convergence theorems on asymptotically demicontractive and hemicontractive mappings in the intermediate sense
}

\section{JO Olaleru and GA Okeke*}

\section{"Correspondence:}

gaokeke1@yahoo.co.uk

Department of Mathematics,

University of Lagos, Akoka, Lagos,

Nigeria

\begin{abstract}
In this study, we introduce two classes of nonlinear mappings, the class of asymptotically demicontractive mappings in the intermediate sense and asymptotically hemicontractive mappings in the intermediate sense and prove the convergence of Mann-type and Ishikawa-type iterative schemes to their respective fixed points. Our results are improvements and generalizations of the results of several authors in the literature.

MSC: $47 \mathrm{H} 10 ; 47 \mathrm{H} 09$

Keywords: asymptotically demicontractive mappings in the intermediate sense; asymptotically hemicontractive mappings in the intermediate sense; Ishikawa iterative scheme; Mann iterative scheme
\end{abstract}

\section{Introduction and preliminaries}

In the sequel, we give the following definitions of some of the concepts that will feature prominently in this study.

We define $C$ as a convex subset of a normed space $E$.

Definition 1.1 Let $T: C \rightarrow C$ be a mapping. $T$ is said to be

(1) asymptotically nonexpansive [1] if there exists a sequence $\left\{k_{n}\right\}$ with $k_{n} \geq 1$ and $\lim k_{n}=$ 1 such that

$$
\left\|T^{n} x-T^{n} y\right\| \leq k_{n}\|x-y\|
$$

for all integers $n \geq 0$ and all $x, y \in C$;

(2) asymptotically strict pseudocontractive [2] if there exist a constant $k \in[0,1)$ and a sequence $\left\{k_{n}\right\} \subset[1, \infty)$ with $k_{n} \rightarrow 1$ as $n \rightarrow \infty$ such that

$$
\left\|T^{n} x-T^{n} y\right\|^{2} \leq k_{n}\|x-y\|^{2}+k\left\|\left(I-T^{n}\right) x-\left(I-T^{n}\right) y\right\|^{2}, \quad \forall x, y \in C .
$$

If $k_{n}=1$ and $T^{n}=T$ for all $n \in \mathbb{N}$ in (1.2), then we obtain the class of strict pseudocontractive mappings. The class of asymptotically strict pseudocontractive mappings was introduced by Qihou in 1987. We remark that the class of asymptotically strict pseudocontractive mappings is a generalization of the class of strict pseudocontractive mappings. Observe that if $k=0$ in (1.2), then we obtain (1.1);

\section{Springer}

(c) 2013 Olaleru and Okeke; licensee Springer. This is an Open Access article distributed under the terms of the Creative Commons Attribution License (http://creativecommons.org/licenses/by/2.0), which permits unrestricted use, distribution, and reproduction in any medium, provided the original work is properly cited. 
(3) asymptotically strict pseudocontractive in the intermediate sense [3] if there exist a constant $k \in[0,1)$ and a sequence $\left\{k_{n}\right\} \subset[1, \infty)$ with $k_{n} \rightarrow 1$ as $n \rightarrow \infty$ such that

$$
\limsup _{n \rightarrow \infty} \sup _{x, y \in C}\left(\left\|T^{n} x-T^{n} y\right\|^{2}-k_{n}\|x-y\|^{2}-k\left\|\left(I-T^{n}\right) x-\left(I-T^{n}\right) y\right\|^{2}\right) \leq 0 .
$$

Put

$$
\zeta_{n}=\max \left\{0, \sup _{x, y \in C}\left(\left\|T^{n} x-T^{n} y\right\|^{2}-k_{n}\|x-y\|^{2}-k\left\|\left(I-T^{n}\right) x-\left(I-T^{n}\right) y\right\|^{2}\right)\right\} .
$$

It follows that $\zeta_{n} \rightarrow 0$ as $n \rightarrow \infty$. Then (1.3) is reduced to the following:

$$
\begin{aligned}
& \left\|T^{n} x-T^{n} y\right\|^{2} \leq k_{n}\|x-y\|^{2}+k\left\|\left(I-T^{n}\right) x-\left(I-T^{n}\right) y\right\|^{2}+\zeta_{n}, \\
& \forall n \geq 1, x, y \in C .
\end{aligned}
$$

We remark that if $\zeta_{n}=0 \forall n \geq 1$ in (1.5), then we obtain (1.2), meaning that the class of asymptotically strict pseudocontractive mappings in the intermediate sense contains properly the class of asymptotically strict pseudocontractive mappings;

(4) asymptotically pseudocontractive [4] if there exists a sequence

$\left\{k_{n}\right\} \subset[1, \infty)$ with $k_{n} \rightarrow 1$ as $n \rightarrow \infty$ such that

$$
\left\langle T^{n} x-T^{n} y, x-y\right\rangle \leq k_{n}\|x-y\|^{2}, \quad \forall n \geq 1, x, y \in C .
$$

It is easy to show that (1.6) is equivalent to

$$
\left\|T^{n} x-T^{n} y\right\|^{2} \leq\left(2 k_{n}-1\right)\|x-y\|^{2}+\left\|x-y-\left(T^{n} x-T^{n} y\right)\right\|^{2}, \quad \forall n \geq 1, x, y \in C .
$$

The class of asymptotically pseudocontractive mappings was introduced in 1991 by Schu [5].

Qin et al. [4] in 2010 introduced the following class of asymptotically pseudocontractive mappings in the intermediate sense. They obtained some weak convergence theorems for this class of nonlinear mappings. They also established a strong convergence theorem without any compact assumption by considering the so-called hybrid projection method;

(5) asymptotically pseudocontractive mapping in the intermediate sense [4] if there exists a sequence $\left\{k_{n}\right\} \subset[1, \infty)$ with $k_{n} \rightarrow 1$ as $n \rightarrow \infty$ such that

$$
\limsup _{n \rightarrow \infty} \sup _{x, y \in C}\left(\left\langle T^{n} x-T^{n} y, x-y\right\rangle-k_{n}\|x-y\|^{2}\right) \leq 0 .
$$

Put

$$
\tau_{n}=\max \left\{0, \sup _{x, y \in C}\left(\left\langle T^{n} x-T^{n} y, x-y\right\rangle-k_{n}\|x-y\|^{2}\right)\right\} .
$$

It follows that $\tau_{n} \rightarrow 0$ as $n \rightarrow \infty$. Hence, (1.8) is reduced to the following:

$$
\left\langle T^{n} x-T^{n} y, x-y\right\rangle \leq k_{n}\|x-y\|^{2}+\tau_{n}, \quad \forall n \geq 1, x, y \in C .
$$


In real Hilbert spaces, it is easy to check that (1.10) is equivalent to

$$
\begin{aligned}
& \left\|T^{n} x-T^{n} y\right\|^{2} \leq\left(2 k_{n}-1\right)\|x-y\|^{2}+\left\|\left(I-T^{n}\right) x-\left(I-T^{n}\right) y\right\|^{2}+2 \tau_{n}, \\
& \forall n \geq 1, x, y \in C .
\end{aligned}
$$

We remark that if $\tau_{n}=0 \forall n \geq 1$, then the class of asymptotically pseudocontractive mappings in the intermediate sense is reduced to the class of asymptotically pseudocontractive mappings;

(6) asymptotically demicontractive mappings [2] if there exists a sequence $\left\{a_{n}\right\}$ such that $\lim _{n} a_{n}=1$ and for $0 \leq k<1$,

$$
\left\|T^{n} x-p\right\|^{2} \leq a_{n}^{2}\|x-p\|^{2}+k\left\|x-T^{n} x\right\|^{2}, \quad \forall n \in \mathbb{N}, x \in C, \forall p \in F(T) .
$$

The class of asymptotically demicontractive maps was introduced in 1987 by Liu [6];

(7) asymptotically hemicontractive mappings [2] if there exists a sequence $\left\{a_{n}\right\}$ such that $\lim _{n} a_{n}=1$ and

$$
\left\|T^{n} x-p\right\|^{2} \leq a_{n}\|x-p\|^{2}+\left\|x-T^{n} x\right\|^{2}, \quad \forall n \in \mathbb{N}, x \in C, \forall p \in F(T) .
$$

The class of asymptotically hemicontractive maps was introduced in 1987 by Liu [6], and it properly contains the class of asymptotically pseudocontractive maps and asymptotically strict pseudocontractive maps in which the fixed point set $F(T):=\{x \in C: T x=x\} \neq \emptyset$ is nonempty. Clearly, if $k=1$ in (1.12), then we obtain (1.13).

Motivated by the above facts, we now introduce the classes of asymptotically demicontractive mappings in the intermediate sense and asymptotically hemicontractive mappings in the intermediate sense as generalizations of the classes of asymptotically demicontractive mappings and asymptotically hemicontractive mappings, respectively.

(8) The map $T: C \rightarrow C$ is said to be an asymptotically demicontractive mapping in the intermediate sense if there exists a sequence $\left\{a_{n}\right\}$ such that $\lim _{n} a_{n}=1$ and for some constant $k \in[0,1)$ if

$$
\begin{aligned}
& \limsup _{n \rightarrow \infty} \sup _{(x, p) \in C \times F(T)}\left(\left\|T^{n} x-p\right\|^{2}-a_{n}^{2}\|x-p\|^{2}-k\left\|x-T^{n} x\right\|^{2}\right) \leq 0, \\
& \forall(x, p) \in C \times F(T) .
\end{aligned}
$$

Observe that if we put

$$
v_{n}=\max \left\{0, \sup _{(x, p) \in C \times F(T)}\left(\left\|T^{n} x-p\right\|^{2}-a_{n}^{2}\|x-p\|^{2}-k\left\|x-T^{n} x\right\|^{2}\right)\right\},
$$

then we get that $v_{n} \longrightarrow 0$ as $n \rightarrow \infty$ and (1.14) is reduced to the following:

$$
\left\|T^{n} x-p\right\|^{2} \leq a_{n}^{2}\|x-p\|^{2}+k\left\|x-T^{n} x\right\|^{2}+v_{n} .
$$

Observe that if $v_{n}=0$ for all $n$ in (1.16), then we obtain (1.12); 
(9) asymptotically hemicontractive mapping in the intermediate sense with sequence $\left\{a_{n}\right\}$ such that $\lim _{n} a_{n}=1$ if

$$
\begin{aligned}
& \limsup _{n \rightarrow \infty} \sup _{(x, p) \in C \times F(T)}\left(\left\|T^{n} x-p\right\|^{2}-a_{n}^{2}\|x-p\|^{2}-\left\|x-T^{n} x\right\|^{2}\right) \leq 0, \\
& \forall(x, p) \in C \times F(T) .
\end{aligned}
$$

Observe that if we put

$$
v_{n}=\max \left\{0, \sup _{(x, p) \in C \times F(T)}\left(\left\|T^{n} x-p\right\|^{2}-a_{n}^{2}\|x-p\|^{2}-\left\|x-T^{n} x\right\|^{2}\right)\right\}
$$

then we get that $v_{n} \longrightarrow 0$ as $n \rightarrow \infty$ and (1.17) is reduced to the following:

$$
\left\|T^{n} x-p\right\|^{2} \leq a_{n}^{2}\|x-p\|^{2}+\left\|x-T^{n} x\right\|^{2}+v_{n} .
$$

Observe that if $v_{n}=0$ for all $n \in \mathbb{N}$ in (1.19), then we obtain (1.13). This means that the class of asymptotically hemicontractive maps in the intermediate sense is a generalization of the class of asymptotically hemicontractive maps. Clearly, if $k=1$ in (1.16), then we obtain (1.19).

The following definition will be useful for our results.

(10) The map $T: C \rightarrow C$ is said to be uniformly L-Lipschitzian [2] if

$$
\left\|T^{n} x-T^{n} y\right\| \leq L\|x-y\|
$$

for some constant $L>0$ for all $n \in \mathbb{N}$ and $x, y \in C$.

Qihou [2] obtained some convergence results of Mann iterative scheme for the class of asymptotically demicontractive mappings. Similarly, Schu [5] proved the convergence of Mann iterative scheme for asymptotically nonexpansive mappings. In this study, we extend the results of Qihou [2] and Schu [5] to the classes of asymptotically demicontractive mappings in the intermediate sense and asymptotically hemicontractive mappings in the intermediate sense. It is our purpose in this study to prove strong convergence theorems of Mann and Ishikawa iterative schemes for uniformly $L$-Lipschitzian asymptotically demicontractive mappings in the intermediate sense and asymptotically hemicontractive maps in the intermediate sense. Our results are extensions and generalizations of the results of Hicks and Kubicek [7], Liu [6], Qihou [2] and Schu [5].

Qihou [2] in 1996 proved the following convergence theorem for the class of asymptotically demicontractive mappings. Cho et al. [8] proved some fixed point theorems for the class of asymptotically demicontractive mappings in arbitrary real normed linear spaces. Maruster and Maruster [9] introduced the class of $\alpha$-demicontractive mappings. They established that this class of nonlinear mappings is general than the class of demicontractive mappings. Olaleru and Mogbademu $[10,11]$ used a three-step iterative scheme to approximate the fixed points of strongly successively pseudocontractive maps.

Theorem Q [2] Let $H$ be a Hilbert space, $C \subset H$ be nonempty closed bounded and convex; $T: C \rightarrow C$ be completely continuous and uniformly L-Lipschitzian and asymptotically 
demicontractive with sequence $\left\{a_{n}\right\}, a_{n} \in[1,+\infty), \sum_{n=0}^{\infty}\left(a_{n}^{2}-1\right)<+\infty, \epsilon \leq \alpha_{n} \leq 1-k-\epsilon$, for $\forall n \in \mathbb{N}$ and some $\epsilon>0, \forall x_{0} \in C$.

$$
x_{n+1}=\alpha_{n} T^{n} x_{n}+\left(1-\alpha_{n}\right) x_{n}, \quad \forall n \in \mathbb{N} .
$$

Then $\left\{x_{n}\right\}_{n=0}^{\infty}$ converges strongly to some fixed point of $T$.

Osilike [12] in 1998 extended the results of Qihou [2] to more general $q$-uniformly smooth Banach spaces, $1<q<\infty$ for the class of asymptotically demicontractive mappings. Osilike and Aniagbosor [13] in 2001 proved that the boundedness requirement imposed on the subset $C$ in the results of Osilike [12] can be dropped. Moore and Nnoli [14] in 2005 proved the necessary and sufficient conditions for the strong convergence of the Mann iterative sequence to a fixed point of an asymptotically demicontractive and uniformly $L$-Lipschitzian map. Zegeye et al. [1] in 2011 obtained some strong convergence results of the Ishikawa-type iterative scheme for the class of asymptotically pseudocontractive mappings in the intermediate sense without resorting to the hybrid method which was the main tool of Qin et al. [4]. Olaleru and Okeke [15] in 2012 established a strong convergence of Noor-type scheme for uniformly $L$-Lipschitzian and asymptotically pseudocontractive mappings in the intermediate sense without assuming any form of compactness. It is our purpose in this paper to prove some strong convergence results using Ishikawa-type and Mann-type iterative schemes for the classes of asymptotically demicontractive mappings in the intermediate sense and asymptotically hemicontractive mappings in the intermediate sense. Our results generalize and improve several other results in literature.

The following lemmas will be useful in this study.

Lemma 1.2 [2] Let sequences $\left\{a_{n}\right\}_{n=1}^{\infty},\left\{b_{n}\right\}_{n=1}^{\infty}$ satisfy $a_{n+1} \leq a_{n}+b_{n}, a_{n} \geq 0, \forall n \in \mathbb{N}, \sum_{n=1}^{\infty} b_{n}$ is convergent and $\left\{a_{n}\right\}_{n=1}^{\infty}$ has a subsequence $\left\{a_{n_{k}}\right\}_{k=1}^{\infty}$ converging to 0 . Then we must have

$$
\lim _{n \rightarrow \infty} a_{n}=0 .
$$

Lemma 1.3 [1] Let $H$ be a real Hilbert space. Then the following equality holds:

$$
\|\alpha x+(1-\alpha) y\|^{2}=\alpha\|x\|^{2}+(1-\alpha)\|y\|^{2}-\alpha(1-\alpha)\|x-y\|^{2}
$$

for all $\alpha \in(0,1)$ and $x, y \in H$.

\section{Main results}

Theorem 2.1 Let $H$ be a Hilbert space, $C \subset H$ be a nonempty closed bounded and convex subset of $H ; T: C \rightarrow C$ be a completely continuous and uniformly L-Lipschitzian and asymptotically demicontractive mapping in the intermediate sense with sequence $\left\{v_{n}\right\}$ as defined in (1.16). Assume that $F(T)$ is nonempty. Let $\left\{x_{n}\right\}$ be a sequence defined by $x_{1}=x \in C$ and

$$
\left\{\begin{array}{l}
y_{n}=\beta_{n} T^{n} x_{n}+\left(1-\beta_{n}\right) x_{n}, \\
x_{n+1}=\alpha_{n} T^{n} y_{n}+\left(1-\alpha_{n}\right) x_{n}, \quad n \geq 1,
\end{array}\right.
$$

where $\left\{\alpha_{n}\right\},\left\{\beta_{n}\right\} \in[0,1]$. Assume that the following conditions are satisfied: 
(i) the sequence $\left\{a_{n}\right\}$ is such that $a_{n} \in[1,+\infty), \forall n \in \mathbb{N}$ and $\sum_{n=1}^{\infty}\left(a_{n}^{2}-1\right)<+\infty$,

(ii) $\sum_{n=0}^{\infty} v_{n}<+\infty$,

(iii) $\epsilon \leq k \leq \alpha_{n} \leq \beta_{n} \leq b \forall n \in \mathbb{N}$ for some $\epsilon>0, k \in[0,1)$ and some $b \in\left(0, L^{-2}\left[\sqrt{1+L^{2}}-1\right]\right)$.

Then $\left\{x_{n}\right\}$ converges strongly to a fixed point of $T$.

Proof Fix $p \in F(T)$. Using (1.16), (2.1) and Lemma 1.3, we obtain

$$
\begin{aligned}
\left\|y_{n}-p\right\|^{2}= & \left\|\beta_{n}\left(T^{n} x_{n}-p\right)+\left(1-\beta_{n}\right)\left(x_{n}-p\right)\right\|^{2} \\
= & \beta_{n}\left\|T^{n} x_{n}-p\right\|^{2}+\left(1-\beta_{n}\right)\left\|x_{n}-p\right\|^{2}-\beta_{n}\left(1-\beta_{n}\right)\left\|T^{n} x_{n}-x_{n}\right\|^{2} \\
\leq & \beta_{n}\left(a_{n}^{2}\left\|x_{n}-p\right\|^{2}+k\left\|x_{n}-T^{n} x_{n}\right\|^{2}+v_{n}\right)+\left(1-\beta_{n}\right)\left\|x_{n}-p\right\|^{2} \\
& -\beta_{n}\left(1-\beta_{n}\right)\left\|T^{n} x_{n}-x_{n}\right\|^{2} \\
= & \beta_{n} a_{n}^{2}\left\|x_{n}-p\right\|^{2}+\beta_{n} k\left\|x_{n}-T^{n} x_{n}\right\|^{2}+\beta_{n} v_{n}+\left(1-\beta_{n}\right)\left\|x_{n}-p\right\|^{2} \\
& -\beta_{n}\left(1-\beta_{n}\right)\left\|T^{n} x_{n}-x_{n}\right\|^{2} \\
= & \left(1+\beta_{n}\left(a_{n}^{2}-1\right)\right)\left\|x_{n}-p\right\|^{2}-\beta_{n}\left(1-\beta_{n}-k\right)\left\|T^{n} x_{n}-x_{n}\right\|^{2} \\
& +\beta_{n} v_{n} .
\end{aligned}
$$

Using (1.20), (2.1) and Lemma 1.3, we have

$$
\begin{aligned}
\left\|y_{n}-T^{n} y_{n}\right\|^{2}= & \left\|\beta_{n}\left(T^{n} x_{n}-T^{n} y_{n}\right)+\left(1-\beta_{n}\right)\left(x_{n}-T^{n} y_{n}\right)\right\|^{2} \\
= & \beta_{n}\left\|T^{n} x_{n}-T^{n} y_{n}\right\|^{2}+\left(1-\beta_{n}\right)\left\|x_{n}-T^{n} y_{n}\right\|^{2} \\
& -\beta_{n}\left(1-\beta_{n}\right)\left\|T^{n} x_{n}-x_{n}\right\|^{2} \\
\leq & \beta_{n} L^{2}\left\|x_{n}-y_{n}\right\|^{2}+\left(1-\beta_{n}\right)\left\|x_{n}-T^{n} y_{n}\right\|^{2} \\
& -\beta_{n}\left(1-\beta_{n}\right)\left\|T^{n} x_{n}-x_{n}\right\|^{2} \\
= & \beta_{n}^{3} L^{2}\left\|x_{n}-T^{n} x_{n}\right\|^{2}+\left(1-\beta_{n}\right)\left\|x_{n}-T^{n} y_{n}\right\|^{2} \\
& -\beta_{n}\left(1-\beta_{n}\right)\left\|T^{n} x_{n}-x_{n}\right\|^{2} .
\end{aligned}
$$

Using (1.16), (2.2) and (2.3), we obtain

$$
\begin{aligned}
\left\|T^{n} y_{n}-p\right\|^{2} \leq & a_{n}^{2}\left\|y_{n}-p\right\|^{2}+k\left\|y_{n}-T^{n} y_{n}\right\|^{2}+v_{n} \\
\leq & a_{n}^{2}\left\{\left(1+\beta_{n}\left(a_{n}^{2}-1\right)\right)\left\|x_{n}-p\right\|^{2}-\beta_{n}\left(1-\beta_{n}-k\right)\left\|T^{n} x_{n}-x_{n}\right\|^{2}+\beta_{n} v_{n}\right\} \\
& +k\left\{\beta_{n}^{3} L^{2}\left\|x_{n}-T^{n} x_{n}\right\|^{2}+\left(1-\beta_{n}\right)\left\|x_{n}-T^{n} y_{n}\right\|^{2}\right. \\
& \left.-\beta_{n}\left(1-\beta_{n}\right)\left\|T^{n} x_{n}-x_{n}\right\|^{2}\right\}+v_{n} \\
= & a_{n}^{2}\left(1+\beta_{n}\left(a_{n}^{2}-1\right)\right)\left\|x_{n}-p\right\|^{2}-a_{n}^{2} \beta_{n}\left(1-\beta_{n}-k\right)\left\|T^{n} x_{n}-x_{n}\right\|^{2} \\
& +a_{n}^{2} \beta_{n} v_{n}+k \beta_{n}^{3} L^{2}\left\|x_{n}-T^{n} x_{n}\right\|^{2}+k\left(1-\beta_{n}\right)\left\|x_{n}-T^{n} y_{n}\right\|^{2} \\
& -k \beta_{n}\left(1-\beta_{n}\right)\left\|T^{n} x_{n}-x_{n}\right\|^{2}+v_{n} .
\end{aligned}
$$


Using (2.4), Lemma 1.3 and condition (iii), we have

$$
\begin{aligned}
\left\|x_{n+1}-p\right\|^{2}= & \left\|\alpha_{n}\left(T^{n} y_{n}-p\right)+\left(1-\alpha_{n}\right)\left(x_{n}-p\right)\right\|^{2} \\
= & \alpha_{n}\left\|T^{n} y_{n}-p\right\|^{2}+\left(1-\alpha_{n}\right)\left\|x_{n}-p\right\|^{2}-\alpha_{n}\left(1-\alpha_{n}\right)\left\|T^{n} y_{n}-x_{n}\right\|^{2} \\
\leq & \alpha_{n}\left\{a_{n}^{2}\left(1+\beta_{n}\left(a_{n}^{2}-1\right)\right)\left\|x_{n}-p\right\|^{2}\right. \\
& -a_{n}^{2} \beta_{n}\left(1-\beta_{n}-k\right)\left\|T^{n} x_{n}-x_{n}\right\|^{2}+a_{n}^{2} \beta_{n} v_{n}+k \beta_{n}^{3} L^{2}\left\|x_{n}-T^{n} x_{n}\right\|^{2} \\
& \left.+k\left(1-\beta_{n}\right)\left\|x_{n}-T^{n} y_{n}\right\|^{2}-k \beta_{n}\left(1-\beta_{n}\right)\left\|T^{n} x_{n}-x_{n}\right\|^{2}+v_{n}\right\} \\
& +\left(1-\alpha_{n}\right)\left\|x_{n}-p\right\|^{2}-\alpha_{n}\left(1-\alpha_{n}\right)\left\|T^{n} y_{n}-x_{n}\right\|^{2} \\
\leq & {\left[1+\alpha_{n}\left(a_{n}^{2}-1\right)\left(1+\beta_{n} a_{n}^{2}\right)\right]\left\|x_{n}-p\right\|^{2} } \\
& -\alpha_{n} \beta_{n}\left[k\left(1-\beta_{n}-\beta_{n}^{2} L^{2}\right)+\left(1-k-\beta_{n}\right) a_{n}^{2}\right]\left\|T^{n} x_{n}-x_{n}\right\|^{2} \\
& +\left[k\left(1-\beta_{n}\right)-\alpha_{n}\left(1-\alpha_{n}\right)\right]\left\|T^{n} y_{n}-x_{n}\right\|^{2}+\alpha_{n}\left(1+a_{n}^{2} \beta_{n}\right) v_{n} \\
\leq & {\left[1+\alpha_{n}\left(a_{n}^{2}-1\right)\left(1+\beta_{n} a_{n}^{2}\right)\right]\left\|x_{n}-p\right\|^{2} } \\
& -\alpha_{n} \beta_{n}\left[k\left(1-\beta_{n}-\beta_{n}^{2} L^{2}\right)+\left(1-k-\beta_{n}\right) a_{n}^{2}\right]\left\|T^{n} x_{n}-x_{n}\right\|^{2} \\
& +\alpha_{n}\left(1+a_{n}^{2} \beta_{n}\right) v_{n} .
\end{aligned}
$$

Observe that by condition (iii), $k\left(1-\beta_{n}\right)-\alpha_{n}\left(1-\alpha_{n}\right)=-M$, where $M>0$, so that the term $\left\|T^{n} y_{n}-x_{n}\right\|^{2}$ can be dropped. Hence, we obtain (2.5).

Next, we show that $\lim _{n \rightarrow \infty}\left\|T^{n} x_{n}-x_{n}\right\|=0$. From (2.5), we have

$$
\begin{aligned}
\left\|x_{n+1}-p\right\|^{2}-\left\|x_{n}-p\right\|^{2} \leq & {\left[\alpha_{n}\left(a_{n}^{2}-1\right)\left(1+\beta_{n} a_{n}^{2}\right)\right]\left\|x_{n}-p\right\|^{2} } \\
& -\alpha_{n} \beta_{n}\left[k\left(1-\beta_{n}-\beta_{n}^{2} L^{2}\right)+\left(1-k-\beta_{n}\right) a_{n}^{2}\right] \\
& \times\left\|T^{n} x_{n}-x_{n}\right\|^{2}+\alpha_{n}\left(1+a_{n}^{2} \beta_{n}\right) v_{n} .
\end{aligned}
$$

Since $\sum_{n=1}^{\infty}\left(a_{n}^{2}-1\right)<+\infty$, it follows that $\lim _{n \rightarrow \infty}\left(a_{n}^{2}-1\right)=0$. Hence, $\left\{a_{n}^{2}\right\}_{n=0}^{\infty}$ is bounded. Since $C$ is bounded and $0 \leq \alpha_{n} \leq \beta_{n} \leq 1,\left\{\alpha_{n}\left(1+\beta_{n} a_{n}^{2}\right)\right\}_{n=1}^{\infty}$ and $\left\{\alpha_{n}\left(1+\beta_{n} a_{n}^{2}\right)\left\|x_{n}-p\right\|^{2}\right\}_{n=1}^{\infty}$ must be bounded. Hence, there exists a constant $M>0$ such that

$$
0 \leq \alpha_{n}\left(1+\beta_{n} a_{n}^{2}\right)\left(1+\left\|x_{n}-p\right\|^{2}\right) \leq M
$$

Using (2.6) and (2.7), we obtain

$$
\begin{aligned}
\left\|x_{n+1}-p\right\|^{2}-\left\|x_{n}-p\right\|^{2} \leq & M\left(a_{n}^{2}-1\right)-\alpha_{n} \beta_{n}\left[k\left(1-\beta_{n}-\beta_{n}^{2} L^{2}\right)\right. \\
& \left.+\left(1-k-\beta_{n}\right) a_{n}^{2}\right]\left\|T^{n} x_{n}-x_{n}\right\|^{2}+M v_{n} .
\end{aligned}
$$

Observe that the condition $b \in\left(0, L^{-2}\left[\sqrt{1+L^{2}}-1\right]\right)$ implies that $b>0$ and $b<L^{-2}\left[\sqrt{1+L^{2}}-\right.$ 1]. This implies that $b L^{2}<\sqrt{1+L^{2}}-1$, hence $1+b L^{2}<\sqrt{1+L^{2}}$. On squaring both sides, we obtain $\left(1+b L^{2}\right)^{2}<\left(\sqrt{1+L^{2}}\right)^{2}$, so that $1+2 b L^{2}+b^{2} L^{4}<1+L^{2}$, so we obtain $L^{2}-2 b L^{2}-b^{2} L^{4}>0$, by dividing through by $L^{2}$, we obtain $1-2 b-b^{2} L^{2}>0$. Hence, $\frac{1-2 b-b^{2} L^{2}}{2}>0$. Since $\lim _{n \rightarrow \infty} a_{n}=1$, there exists a natural number $N$ such that for $n>N$,

$$
k\left(1-\beta_{n}-\beta_{n}^{2} L^{2}\right)+\left(1-k-\beta_{n}\right) a_{n}^{2} \geq 1-b-a_{n}^{2} b-L^{2} b^{2} \geq \frac{1-2 b-b^{2} L^{2}}{2} .
$$


Assuming that $\lim _{n \rightarrow \infty}\left\|T^{n} x_{n}-x_{n}\right\| \neq 0$, then there exist $\epsilon_{0}>0$ and a subsequence $\left\{x_{n_{r}}\right\}_{r=1}^{\infty}$ of $\left\{x_{n}\right\}_{n=1}^{\infty}$ such that

$$
\left\|x_{n_{r}}-T^{n_{r}} x_{n_{r}}\right\|^{2} \geq \epsilon_{0}
$$

Without loss of generality, we can assume that $n_{1}>N$. From (2.8), we obtain

$$
\begin{gathered}
\alpha_{n} \beta_{n}\left[k\left(1-\beta_{n}-\beta_{n}^{2} L^{2}\right)+\left(1-k-\beta_{n}\right) a_{n}^{2}\right]\left\|T^{n} x_{n}-x_{n}\right\|^{2} \\
\leq M\left(a_{n}^{2}-1\right)+\left\|x_{n}-p\right\|^{2}-\left\|x_{n+1}-p\right\|^{2}+M v_{n} .
\end{gathered}
$$

Hence,

$$
\begin{aligned}
& \sum_{m=n_{1}}^{n_{r}} \alpha_{m} \beta_{m}\left[k\left(1-\beta_{m}-\beta_{m}^{2} L^{2}\right)+\left(1-k-\beta_{m}\right) a_{m}^{2}\right]\left\|T^{m} x_{m}-x_{m}\right\|^{2} \\
& \leq \sum_{m=n_{1}}^{n_{r}} M\left(a_{m}^{2}-1\right)+\left\|x_{n_{1}}-p\right\|^{2}-\left\|x_{n_{r}+1}-p\right\|^{2}+\sum_{m=n_{1}}^{n_{r}} M v_{m}, \\
& \sum_{l=1}^{r} \alpha_{n_{l}} \beta_{n_{l}}\left[k\left(1-\beta_{n_{l}}-\beta_{n_{l}} L^{2}\right)+\left(1-k-\beta_{n_{l}}\right) a_{n_{l}}^{2}\right]\left\|T^{n_{l}} x_{n_{l}}-x_{n_{l}}\right\|^{2} \\
& \quad \leq \sum_{m=n_{1}}^{n_{r}} M\left(a_{m}^{2}-1\right)+\left\|x_{n_{1}}-p\right\|^{2}-\left\|x_{n_{r}+1}-p\right\|^{2}+\sum_{m=n_{1}}^{n_{r}} M v_{m} .
\end{aligned}
$$

From (2.9), (2.10), (2.11) and $0 \leq \epsilon \leq \alpha_{n} \leq \beta_{n}$, we observe that

$$
\begin{aligned}
& r \epsilon^{2}\left(\frac{1-2 b-b^{2} L^{2}}{2}\right) \epsilon_{0} \\
& \quad \leq \sum_{m=n_{l}}^{n_{r}} M\left(a_{m}^{2}-1\right)+\left\|x_{n_{1}}-p\right\|^{2}-\left\|x_{n_{r}+1}-p\right\|^{2}+\sum_{m=n_{l}}^{n_{r}} M v_{m} .
\end{aligned}
$$

From $\sum_{n=1}^{\infty}\left(a_{n}^{2}-1\right)<+\infty, \sum_{n=1}^{\infty} v_{n}<+\infty$ and the boundedness of $C$, we observe that the right-hand side of (2.12) is bounded. However, the left-hand side of (2.12) is positively unbounded when $r \rightarrow \infty$. Hence, a contradiction. Therefore

$$
\lim _{n \rightarrow \infty}\left\|x_{n}-T^{n} x_{n}\right\|=0
$$

Using (2.1), we have

$$
\begin{aligned}
\left\|x_{n+1}-x_{n}\right\| & =\left\|\alpha_{n} T^{n} y_{n}+\left(1-\alpha_{n}\right) x_{n}-x_{n}\right\| \\
& =\left\|\alpha_{n}\left(T^{n} y_{n}-x_{n}\right)\right\| \\
& \leq \alpha_{n}\left\|T^{n} y_{n}-x_{n}\right\| \\
& \leq \alpha_{n}\left(\left\|T^{n} y_{n}-T^{n} x_{n}\right\|+\left\|T^{n} x_{n}-x_{n}\right\|\right) \\
& \leq \alpha_{n}\left(L\left\|y_{n}-x_{n}\right\|+\left\|T^{n} x_{n}-x_{n}\right\|\right) \\
& \leq \alpha_{n}\left(1+\beta_{n} L\right)\left\|T^{n} x_{n}-x_{n}\right\| .
\end{aligned}
$$


Using (2.13), we obtain

$$
\lim _{n \rightarrow \infty}\left\|x_{n+1}-x_{n}\right\|=0
$$

Observe that

$$
\begin{aligned}
\left\|x_{n}-T x_{n}\right\| \leq & \left\|x_{n}-x_{n+1}\right\|+\left\|x_{n+1}-T^{n+1} x_{n+1}\right\|+\left\|T^{n+1} x_{n+1}-T^{n+1} x_{n}\right\| \\
& +\left\|T^{n+1} x_{n}-T x_{n}\right\| \\
\leq & (1+L)\left\|x_{n}-x_{n+1}\right\|+\left\|x_{n+1}-T^{n+1} x_{n+1}\right\|+L\left\|T^{n} x_{n}-x_{n}\right\| .
\end{aligned}
$$

Using (2.13) and (2.15), we have

$$
\lim _{n \rightarrow \infty}\left\|x_{n}-T x_{n}\right\|=0
$$

Since $\left\{x_{n}\right\}$ is bounded, the sequence $\left\{T x_{n}\right\}$ has a convergent subsequence $\left\{T x_{n_{r}}\right\}$ say. Let $T x_{n_{r}} \rightarrow q$ as $r \rightarrow \infty$. Then $x_{n_{r}} \rightarrow q$ as $r \rightarrow \infty$ since

$$
0=\lim _{r \rightarrow \infty}\left[x_{n_{r}}-T x_{n_{r}}\right]=\lim _{r \rightarrow \infty} x_{n_{r}}-\lim _{r \rightarrow \infty} T x_{n_{r}}=\lim _{r \rightarrow \infty} x_{n_{r}}-q .
$$

By the continuity of $T, T x_{n_{r}} \rightarrow T q$ as $r \rightarrow \infty$ but $T x_{n_{r}} \rightarrow q$ as $r \rightarrow \infty$. Hence, $q=T q$.

Hence, $\left\{x_{n}\right\}_{n=0}^{\infty}$ has a subsequence which converges to the fixed point $q$ of $T$. Using (2.9), there exists some natural number $N$, when $n>N, k\left(1-\beta_{n}-\beta_{n}^{2} L^{2}\right)+\left(1-k-\beta_{n}\right) a_{n}^{2} \geq$ $\frac{1-2 b-b^{2} L^{2}}{2}>0 \forall n>N$. Using (2.7), $0 \leq \alpha_{n}\left(1+\beta_{n} a_{n}^{2}\right) v_{n}+\alpha_{n}\left(1+\beta_{n} a_{n}^{2}\right)\left(a_{n}^{2}-1\right)\left\|x_{n}-q\right\|^{2} \leq$ $M\left(a_{n}^{2}-1\right)+M v_{n}$. From (2.6),

$$
\left\|x_{n+1}-q\right\|^{2} \leq\left\|x_{n}-q\right\|^{2}+M\left(a_{n}^{2}-1+v_{n}\right)
$$

But $\sum_{n=1}^{\infty}\left(a_{n}^{2}-1\right)<+\infty$ and $\sum_{n=1}^{\infty} v_{n}<+\infty$ imply that $\sum_{n=1}^{\infty} M\left(a_{n}^{2}-1+v_{n}\right)<+\infty$. From (2.18), it follows that there exists a subsequence $\left\{\left\|x_{n_{r}}-q\right\|^{2}\right\}_{r=1}^{\infty}$ of $\left\{\left\|x_{n}-q\right\|^{2}\right\}_{n=1}^{\infty}$, which converges to 0 . Hence, using (2.19) and Lemma $1.2, \lim _{n \rightarrow \infty}\left\|x_{n}-q\right\|^{2}=0$. This means that $\lim _{n \rightarrow \infty} x_{n}=q$. The proof of the theorem is complete.

Remark 2.2 Theorem 2.1 extends the results of Osilike [12], Osilike and Aniagbosor [13], Igbokwe [16] in the framework of Hilbert spaces since the class of asymptotically demicontractive maps considered by these authors is a subclass of the class of asymptotically demicontractive maps in the intermediate sense introduced in this article.

Theorem 2.3 Let $H$ be a Hilbert space, $C \subset H$ be a nonempty closed bounded and convex subset of $H ; T: C \rightarrow C$ be a completely continuous and asymptotically demicontractive mapping in the intermediate sense with sequence $\left\{v_{n}\right\}$ as defined in (1.16). Assume that $F(T)$ is nonempty. Let $\left\{x_{n}\right\}$ be a sequence defined by $x_{0}=x \in C$ and

$$
x_{n+1}=\alpha_{n} T^{n} x_{n}+\left(1-\alpha_{n}\right) x_{n}, \quad \forall n \in \mathbb{N},
$$

where $\alpha_{n} \in[0,1]$. Assume that the following conditions are satisfied:

(i) the sequence $\left\{a_{n}\right\}$ is such that $a_{n} \in[1,+\infty)$ and $\sum_{n=0}^{\infty}\left(a_{n}^{2}-1\right)<+\infty$, 
(ii) $\sum_{n=0}^{\infty} v_{n}<+\infty$ and

(iii) $\epsilon \leq \alpha_{n} \leq 1-k-\epsilon \forall n \in \mathbb{N}$ for some $\epsilon>0$ and $k \in[0,1)$.

Then $\left\{x_{n}\right\}_{n=0}^{\infty}$ converges strongly to a fixed point of $T$.

Proof Using (1.16), we obtain

$$
\left\|T^{n} x_{n}-p\right\|^{2} \leq a_{n}^{2}\left\|x_{n}-p\right\|^{2}+k\left\|x_{n}-T^{n} x_{n}\right\|^{2}+v_{n} .
$$

From (2.21) and Lemma 1.3, we have

$$
\begin{aligned}
\left\|x_{n+1}-p\right\|^{2}= & \left\|\alpha_{n}\left(T^{n} x_{n}-p\right)+\left(1-\alpha_{n}\right)\left(x_{n}-p\right)\right\|^{2} \\
= & \alpha_{n}\left\|T^{n} x_{n}-p\right\|^{2}+\left(1-\alpha_{n}\right)\left\|x_{n}-p\right\|^{2} \\
& -\alpha_{n}\left(1-\alpha_{n}\right)\left\|T^{n} x_{n}-x_{n}\right\|^{2} \\
\leq & \alpha_{n}\left(a_{n}^{2}\left\|x_{n}-p\right\|^{2}+k\left\|x_{n}-T^{n} x_{n}\right\|^{2}+v_{n}\right)+\left(1-\alpha_{n}\right)\left\|x_{n}-p\right\|^{2} x \\
& -\alpha_{n}\left(1-\alpha_{n}\right)\left\|T^{n} x_{n}-x_{n}\right\|^{2} \\
= & \alpha_{n} a_{n}^{2}\left\|x_{n}-p\right\|^{2}+\alpha_{n} k\left\|x_{n}-T^{n} x_{n}\right\|^{2}+\alpha_{n} v_{n}+\left(1-\alpha_{n}\right)\left\|x_{n}-p\right\|^{2} \\
& -\alpha_{n}\left(1-\alpha_{n}\right)\left\|T^{n} x_{n}-x_{n}\right\|^{2} \\
= & \left\|x_{n}-p\right\|^{2}+\alpha_{n}\left(a_{n}^{2}-1\right)\left\|x_{n}-p\right\|^{2} \\
& -\alpha_{n}\left(1-\alpha_{n}-k\right)\left\|T^{n} x_{n}-x_{n}\right\|^{2}+\alpha_{n} v_{n} .
\end{aligned}
$$

Now, we show that $\lim _{n \rightarrow \infty}\left\|T^{n} x_{n}-x_{n}\right\|=0$. But $0<\epsilon \leq \alpha_{n} \leq 1-k-\epsilon, 1-k-\alpha_{n} \geq \epsilon$. Hence $\alpha_{n}\left(1-k-\alpha_{n}\right) \geq \epsilon^{2}$ and $v_{n} \longrightarrow 0$ as $n \rightarrow \infty$. From (2.22), we have

$$
\left\|x_{n+1}-p\right\|^{2} \leq\left\|x_{n}-p\right\|^{2}+\alpha_{n}\left(a_{n}^{2}-1\right)\left\|x_{n}-p\right\|^{2}-\epsilon^{2}\left\|T^{n} x_{n}-x_{n}\right\|^{2}+\alpha_{n} v_{n} .
$$

Since $C$ is bounded and $T$ is a self-mapping on $C$, it follows that there exists some $M>0$ such that $\left\|x_{n}-p\right\|^{2} \leq M, \forall n \in \mathbb{N}$. But $\alpha_{n} \in[0,1]$, from (2.23) we obtain

$$
\left\|x_{n+1}-p\right\|^{2} \leq\left\|x_{n}-p\right\|^{2}+M\left(a_{n}^{2}-1\right)+v_{n}-\epsilon^{2}\left\|T^{n} x_{n}-x_{n}\right\|^{2} .
$$

Hence,

$$
\begin{aligned}
& \epsilon^{2}\left\|T^{n} x_{n}-x_{n}\right\|^{2} \leq\left\|x_{n}-p\right\|^{2}+M\left(a_{n}^{2}-1\right)+v_{n}-\left\|x_{n+1}-p\right\|^{2}, \\
& \sum_{n=1}^{m} \epsilon^{2}\left\|T^{n} x_{n}-x_{n}\right\|^{2} \leq\left\|x_{1}-p\right\|^{2}-\left\|x_{m+1}-p\right\|^{2}+\sum_{n=1}^{m}\left[M\left(a_{n}^{2}-1\right)+v_{n}\right] \\
& \leq 2 M+\sum_{n=1}^{\infty}\left[M\left(a_{n}^{2}-1\right)+v_{n}\right] .
\end{aligned}
$$

But $\sum_{n=1}^{\infty}\left[M\left(a_{n}^{2}-1\right)+v_{n}\right]<+\infty, \sum_{n=1}^{\infty} \epsilon^{2}\left\|T^{n} x_{n}-x_{n}\right\|^{2}<+\infty$. Hence, we obtain $\lim _{n \rightarrow \infty}\left\|T^{n} x_{n}-x_{n}\right\|^{2}=0$. So that

$$
\lim _{n \rightarrow \infty}\left\|T^{n} x_{n}-x_{n}\right\|=0
$$


Since $\left\{x_{n}\right\}_{n=0}^{\infty}$ is a bounded sequence and $T$ is completely continuous, hence there is a subsequence $\left\{T x_{n_{k}}\right\}_{k=0}^{\infty}$ of $\left\{T x_{n}\right\}_{n=0}^{\infty}$. Using (2.27), $\left\{x_{n}\right\}_{n=0}^{\infty}$ must have a convergent subsequence $\left\{x_{n_{k}}\right\}_{k=0}^{\infty}$. Assume $\lim _{k \rightarrow \infty} x_{n_{k}}=x^{*}$. From the continuity of $T$ and using (2.27), we obtain $x^{*}=T x^{*}$, meaning that $x^{*}$ is a fixed point of $T$. Hence, $\left\{x_{n}\right\}_{n=0}^{\infty}$ has a subsequence which converges to a fixed point $x^{*}$ of $T$.

Since $\sum_{n=1}^{\infty} \epsilon^{2}\left\|T^{n} x_{n}-x_{n}\right\|^{2}<+\infty$ and $\sum_{n=1}^{\infty}\left[M\left(a_{n}^{2}-1\right)+v_{n}\right]<+\infty$ and using Lemma 1.2, we obtain

$$
\lim _{n \rightarrow \infty}\left\|x_{n}-x^{*}\right\|^{2}=0
$$

Hence, $\lim _{n \rightarrow \infty} x_{n}=x^{*}$. The proof of Theorem 2.3 is completed.

Corollary 2.4 Let $H$ be a Hilbert space, $C \subset H$ be a nonempty closed bounded and convex subset of $H ; T: C \rightarrow C$ be a completely continuous and uniformly L-Lipschitzian and asymptotically demicontractive mapping with sequence $\left\{a_{n}\right\}$ as defined in (1.12). Assume that $F(T)$ is nonempty. Let $\left\{x_{n}\right\}$ be a sequence defined by $x_{0}=x \in C$ and

$$
x_{n+1}=\alpha_{n} T^{n} x_{n}+\left(1-\alpha_{n}\right) x_{n}, \quad \forall n \in \mathbb{N},
$$

where $\alpha_{n} \in[0,1]$. Assume that the following conditions are satisfied:

(i) the sequence $\left\{a_{n}\right\}$ is such that $a_{n} \in[1,+\infty)$ and $\sum_{n=0}^{\infty}\left(a_{n}^{2}-1\right)<+\infty$ and

(ii) $\epsilon \leq \alpha_{n} \leq 1-k-\epsilon, \forall n \in \mathbb{N}, k \in[0,1)$ and some $\epsilon>0$.

Then $\left\{x_{n}\right\}_{n=0}^{\infty}$ converges strongly to a fixed point of $T$.

Remark 2.5 Corollary 2.4 is Theorem 1 of Qihou [2] when $v_{n}=0$ for all $n \in \mathbb{N}$ in Theorem 2.3.

Theorem 2.6 Let $H$ be a Hilbert space, $C \subset H$ be a nonempty closed bounded and convex subset of $H ; T: C \rightarrow C$ be a completely continuous and uniformly L-Lipschitzian and asymptotically hemicontractive mapping in the intermediate sense with sequence $\left\{v_{n}\right\}$ as defined in (1.19). Assume that $F(T)$ is nonempty. Let $\left\{x_{n}\right\}$ be a sequence defined by $x_{1}=x \in C$ and

$$
\left\{\begin{array}{l}
y_{n}=\beta_{n} T^{n} x_{n}+\left(1-\beta_{n}\right) x_{n}, \\
x_{n+1}=\alpha_{n} T^{n} y_{n}+\left(1-\alpha_{n}\right) x_{n}, \quad n \geq 1,
\end{array}\right.
$$

where $\alpha_{n}, \beta_{n} \in[0,1]$. Assume that the following conditions are satisfied:

(i) the sequence $\left\{a_{n}\right\}$ is such that $a_{n} \in[1,+\infty) \forall n \in \mathbb{N}$ and $\sum_{n=1}^{\infty}\left(a_{n}^{2}-1\right)<+\infty$,

(ii) $\sum_{n=0}^{\infty} v_{n}<+\infty$,

(iii) $\epsilon \leq \alpha_{n} \leq \beta_{n} \leq b \forall n \in \mathbb{N}$ for some $\epsilon>0$ and some $b \in\left(0, L^{-2}\left[\sqrt{1+L^{2}}-1\right]\right)$.

Then $\left\{x_{n}\right\}$ converges strongly to a fixed point of $T$.

Proof Fix $p \in F(T)$. Using (1.19), (2.30) and Lemma 1.3, we obtain

$$
\begin{aligned}
\left\|y_{n}-p\right\|^{2} & =\left\|\beta_{n}\left(T^{n} x_{n}-p\right)+\left(1-\beta_{n}\right)\left(x_{n}-p\right)\right\|^{2} \\
& =\beta_{n}\left\|T^{n} x_{n}-p\right\|^{2}+\left(1-\beta_{n}\right)\left\|x_{n}-p\right\|^{2}-\beta_{n}\left(1-\beta_{n}\right)\left\|T^{n} x_{n}-x_{n}\right\|^{2}
\end{aligned}
$$




$$
\begin{aligned}
\leq & \beta_{n}\left(a_{n}^{2}\left\|x_{n}-p\right\|^{2}+\left\|x_{n}-T^{n} x_{n}\right\|^{2}+v_{n}\right)+\left(1-\beta_{n}\right)\left\|x_{n}-p\right\|^{2} \\
& -\beta_{n}\left(1-\beta_{n}\right)\left\|T^{n} x_{n}-x_{n}\right\|^{2} \\
= & \beta_{n} a_{n}^{2}\left\|x_{n}-p\right\|^{2}+\beta_{n}\left\|x_{n}-T^{n} x_{n}\right\|^{2}+\beta_{n} v_{n}+\left(1-\beta_{n}\right)\left\|x_{n}-p\right\|^{2} \\
& -\beta_{n}\left(1-\beta_{n}\right)\left\|T^{n} x_{n}-x_{n}\right\|^{2} \\
= & \left(1+\beta_{n}\left(a_{n}^{2}-1\right)\right)\left\|x_{n}-p\right\|^{2}+\beta_{n}^{2}\left\|T^{n} x_{n}-x_{n}\right\|^{2} \\
& +\beta_{n} v_{n} .
\end{aligned}
$$

Using (1.20), (2.30) and Lemma 1.3, we have

$$
\begin{aligned}
\left\|y_{n}-T^{n} y_{n}\right\|^{2}= & \left\|\beta_{n}\left(T^{n} x_{n}-T^{n} y_{n}\right)+\left(1-\beta_{n}\right)\left(x_{n}-T^{n} y_{n}\right)\right\|^{2} \\
= & \beta_{n}\left\|T^{n} x_{n}-T^{n} y_{n}\right\|^{2}+\left(1-\beta_{n}\right)\left\|x_{n}-T^{n} y_{n}\right\|^{2} \\
& -\beta_{n}\left(1-\beta_{n}\right)\left\|T^{n} x_{n}-x_{n}\right\|^{2} \\
\leq & \beta_{n} L^{2}\left\|x_{n}-y_{n}\right\|^{2}+\left(1-\beta_{n}\right)\left\|x_{n}-T^{n} y_{n}\right\|^{2} \\
& -\beta_{n}\left(1-\beta_{n}\right)\left\|T^{n} x_{n}-x_{n}\right\|^{2} \\
= & \beta_{n}^{3} L^{2}\left\|x_{n}-T^{n} x_{n}\right\|^{2}+\left(1-\beta_{n}\right)\left\|x_{n}-T^{n} y_{n}\right\|^{2} \\
& -\beta_{n}\left(1-\beta_{n}\right)\left\|T^{n} x_{n}-x_{n}\right\|^{2} .
\end{aligned}
$$

Using (1.19), (2.31) and (2.32), we obtain

$$
\begin{aligned}
\left\|T^{n} y_{n}-p\right\|^{2} \leq & a_{n}^{2}\left\|y_{n}-p\right\|^{2}+\left\|y_{n}-T^{n} y_{n}\right\|^{2}+v_{n} \\
\leq & a_{n}^{2}\left\{\left(1+\beta_{n}\left(a_{n}^{2}-1\right)\right)\left\|x_{n}-p\right\|^{2}+\beta_{n}^{2}\left\|T^{n} x_{n}-x_{n}\right\|^{2}+\beta_{n} v_{n}\right\} \\
& +\beta_{n}^{3} L^{2}\left\|x_{n}-T^{n} x_{n}\right\|^{2}+\left(1-\beta_{n}\right)\left\|x_{n}-T^{n} y_{n}\right\|^{2} \\
& -\beta_{n}\left(1-\beta_{n}\right)\left\|T^{n} x_{n}-x_{n}\right\|^{2}+v_{n} \\
= & a_{n}^{2}\left(1+\beta_{n}\left(a_{n}^{2}-1\right)\right)\left\|x_{n}-p\right\|^{2}+a_{n}^{2} \beta_{n}^{2}\left\|T^{n} x_{n}-x_{n}\right\|^{2} \\
& +a_{n}^{2} \beta_{n} v_{n}+\beta_{n}^{3} L^{2}\left\|x_{n}-T^{n} x_{n}\right\|^{2}+\left(1-\beta_{n}\right)\left\|x_{n}-T^{n} y_{n}\right\|^{2} \\
& -\beta_{n}\left(1-\beta_{n}\right)\left\|T^{n} x_{n}-x_{n}\right\|^{2}+v_{n} .
\end{aligned}
$$

Using (2.33), Lemma 1.3 and condition (iii), we have

$$
\begin{aligned}
\left\|x_{n+1}-p\right\|^{2}= & \left\|\alpha_{n}\left(T^{n} y_{n}-p\right)+\left(1-\alpha_{n}\right)\left(x_{n}-p\right)\right\|^{2} \\
= & \alpha_{n}\left\|T^{n} y_{n}-p\right\|^{2}+\left(1-\alpha_{n}\right)\left\|x_{n}-p\right\|^{2}-\alpha_{n}\left(1-\alpha_{n}\right)\left\|T^{n} y_{n}-x_{n}\right\|^{2} \\
\leq & \alpha_{n}\left\{a_{n}^{2}\left(1+\beta_{n}\left(a_{n}^{2}-1\right)\right)\left\|x_{n}-p\right\|^{2}\right. \\
& +a_{n}^{2} \beta_{n}^{2}\left\|T^{n} x_{n}-x_{n}\right\|^{2}+a_{n}^{2} \beta_{n} v_{n}+\beta_{n}^{3} L^{2}\left\|x_{n}-T^{n} x_{n}\right\|^{2} \\
& \left.+\left(1-\beta_{n}\right)\left\|x_{n}-T^{n} y_{n}\right\|^{2}-\beta_{n}\left(1-\beta_{n}\right)\left\|T^{n} x_{n}-x_{n}\right\|^{2}+v_{n}\right\} \\
& +\left(1-\alpha_{n}\right)\left\|x_{n}-p\right\|^{2}-\alpha_{n}\left(1-\alpha_{n}\right)\left\|T^{n} y_{n}-x_{n}\right\|^{2} \\
\leq & {\left[1+\alpha_{n}\left(a_{n}^{2}-1\right)\left(1+\beta_{n} a_{n}^{2}\right)\right]\left\|x_{n}-p\right\|^{2} }
\end{aligned}
$$




$$
\begin{aligned}
& -\alpha_{n} \beta_{n}\left[1-\beta_{n}-\beta_{n}^{2} L^{2}-\beta_{n} a_{n}^{2}\right]\left\|T^{n} x_{n}-x_{n}\right\|^{2} \\
& +\left[\left(1-\beta_{n}\right)-\alpha_{n}\left(1-\alpha_{n}\right)\right]\left\|T^{n} y_{n}-x_{n}\right\|^{2}+\alpha_{n}\left(1+a_{n}^{2} \beta_{n}\right) v_{n} \\
\leq & {\left[1+\alpha_{n}\left(a_{n}^{2}-1\right)\left(1+\beta_{n} a_{n}^{2}\right)\right]\left\|x_{n}-p\right\|^{2} } \\
& -\alpha_{n} \beta_{n}\left[1-\beta_{n}-\beta_{n}^{2} L^{2}-\beta_{n} a_{n}^{2}\right]\left\|T^{n} x_{n}-x_{n}\right\|^{2} \\
& +\alpha_{n}\left(1+a_{n}^{2} \beta_{n}\right) v_{n} .
\end{aligned}
$$

Next, we show that $\lim _{n \rightarrow \infty}\left\|T^{n} x_{n}-x_{n}\right\|=0$. From (2.34), we have

$$
\begin{aligned}
\left\|x_{n+1}-p\right\|^{2}-\left\|x_{n}-p\right\|^{2} \leq & {\left[\alpha_{n}\left(a_{n}^{2}-1\right)\left(1+\beta_{n} a_{n}^{2}\right)\right]\left\|x_{n}-p\right\|^{2} } \\
& -\alpha_{n} \beta_{n}\left[1-\beta_{n}-\beta_{n}^{2} L^{2}-\beta_{n} a_{n}^{2}\right] \\
& \times\left\|T^{n} x_{n}-x_{n}\right\|^{2}+\alpha_{n}\left(1+a_{n}^{2} \beta_{n}\right) v_{n} .
\end{aligned}
$$

Since $\sum_{n=1}^{\infty}\left(a_{n}^{2}-1\right)<+\infty$, it follows that $\lim _{n \rightarrow \infty}\left(a_{n}^{2}-1\right)=0$. Hence, $\left\{a_{n}^{2}\right\}_{n=0}^{\infty}$ is bounded. Since $C$ is bounded and $0 \leq \alpha_{n} \leq \beta_{n} \leq 1,\left\{\alpha_{n}\left(1+\beta_{n} a_{n}^{2}\right)\right\}_{n=1}^{\infty}$ and $\left\{\alpha_{n}\left(1+\beta_{n} a_{n}^{2}\right)\left\|x_{n}-p\right\|^{2}\right\}_{n=1}^{\infty}$ must be bounded. Hence, there exists a constant $M>0$ such that

$$
0 \leq \alpha_{n}\left(1+\beta_{n} a_{n}^{2}\right)\left(1+\left\|x_{n}-p\right\|^{2}\right) \leq M
$$

Using (2.35) and (2.36), we obtain

$$
\begin{aligned}
\left\|x_{n+1}-p\right\|^{2}-\left\|x_{n}-p\right\|^{2} \leq & M\left(a_{n}^{2}-1\right)-\alpha_{n} \beta_{n}\left[1-\beta_{n}-\beta_{n}^{2} L^{2}-\beta_{n} a_{n}^{2}\right] \\
& \times\left\|T^{n} x_{n}-x_{n}\right\|^{2}+M v_{n} .
\end{aligned}
$$

Observe that the condition $b \in\left(0, L^{-2}\left[\sqrt{1+L^{2}}-1\right]\right)$ implies that $b>0$ and $b<L^{-2}\left[\sqrt{1+L^{2}}-\right.$ 1]. This implies that $b L^{2}<\sqrt{1+L^{2}}-1$, hence $1+b L^{2}<\sqrt{1+L^{2}}$. On squaring both sides, we obtain $\left(1+b L^{2}\right)^{2}<\left(\sqrt{1+L^{2}}\right)^{2}$, so that $1+2 b L^{2}+b^{2} L^{4}<1+L^{2}$, so we obtain $L^{2}-2 b L^{2}-b^{2} L^{4}>0$, by dividing through by $L^{2}$, we obtain $1-2 b-b^{2} L^{2}>0$. Hence, $\frac{1-2 b-b^{2} L^{2}}{2}>0$. Since $\lim _{n \rightarrow \infty} a_{n}=1$, there exists a natural number $N$ such that for $n>N$,

$$
1-\beta_{n}-\beta_{n}^{2} L^{2}-\beta_{n} a_{n}^{2} \geq 1-b-a_{n}^{2} b-L^{2} b^{2} \geq \frac{1-2 b-b^{2} L^{2}}{2} .
$$

Assuming that $\lim _{n \rightarrow \infty}\left\|T^{n} x_{n}-x_{n}\right\| \neq 0$, then there exist $\epsilon_{0}>0$ and a subsequence $\left\{x_{n_{r}}\right\}_{r=1}^{\infty}$ of $\left\{x_{n}\right\}_{n=1}^{\infty}$ such that

$$
\left\|x_{n_{r}}-T^{n_{r}} x_{n_{r}}\right\|^{2} \geq \epsilon_{0}
$$

Without loss of generality, we can assume that $n_{1}>N$. From (2.37), we obtain

$$
\begin{aligned}
& \alpha_{n} \beta_{n}\left[1-\beta_{n}-\beta_{n}^{2} L^{2}-\beta_{n} a_{n}^{2}\right]\left\|T^{n} x_{n}-x_{n}\right\|^{2} \\
& \quad \leq M\left(a_{n}^{2}-1\right)+\left\|x_{n}-p\right\|^{2}-\left\|x_{n+1}-p\right\|^{2}+M v_{n} .
\end{aligned}
$$


Hence,

$$
\begin{aligned}
& \sum_{m=n_{1}}^{n_{r}} \alpha_{m} \beta_{m}\left[1-\beta_{m}-\beta_{m}^{2} L^{2}-\beta_{m} a_{m}^{2}\right]\left\|T^{m} x_{m}-x_{m}\right\|^{2} \\
& \quad \leq \sum_{m=n_{1}}^{n_{r}} M\left(a_{m}^{2}-1\right)+\left\|x_{n_{1}}-p\right\|^{2}-\left\|x_{n_{r}+1}-p\right\|^{2}+\sum_{m=n_{1}}^{n_{r}} M v_{m}, \\
& \sum_{l=1}^{r} \alpha_{n_{l}} \beta_{n_{l}}\left[1-\beta_{n_{l}}-\beta_{n_{l}} L^{2}-\beta_{n_{l}} a_{n_{l}}^{2}\right]\left\|T^{n_{l}} x_{n_{l}}-x_{n_{l}}\right\|^{2} \\
& \quad \leq \sum_{m=n_{1}}^{n_{r}} M\left(a_{m}^{2}-1\right)+\left\|x_{n_{1}}-p\right\|^{2}-\left\|x_{n_{r}+1}-p\right\|^{2}+\sum_{m=n_{1}}^{n_{r}} M v_{m} .
\end{aligned}
$$

From (2.38), (2.39), (2.40) and $0 \leq \epsilon \leq \alpha_{n} \leq \beta_{n}$, we observe that

$$
\begin{aligned}
& r \epsilon^{2}\left(\frac{1-2 b-b^{2} L^{2}}{2}\right) \epsilon_{0} \\
& \quad \leq \sum_{m=n_{l}}^{n_{r}} M\left(a_{m}^{2}-1\right)+\left\|x_{n_{1}}-p\right\|^{2}-\left\|x_{n_{r}+1}-p\right\|^{2}+\sum_{m=n_{l}}^{n_{r}} M v_{m}
\end{aligned}
$$

From $\sum_{n=1}^{\infty}\left(a_{n}^{2}-1\right)<+\infty, \sum_{n=1}^{\infty} v_{n}<+\infty$ and the boundedness of $C$, we observe that the right-hand side of (2.41) is bounded. However, the left-hand side of (2.41) is positively unbounded when $r \rightarrow \infty$. Hence, a contradiction. Therefore

$$
\lim _{n \rightarrow \infty}\left\|x_{n}-T^{n} x_{n}\right\|=0
$$

Using (2.30), we have

$$
\begin{aligned}
\left\|x_{n+1}-x_{n}\right\| & =\left\|\alpha_{n} T^{n} y_{n}+\left(1-\alpha_{n}\right) x_{n}-x_{n}\right\| \\
& =\left\|\alpha_{n}\left(T^{n} y_{n}-x_{n}\right)\right\| \\
& \leq \alpha_{n}\left\|T^{n} y_{n}-x_{n}\right\| \\
& \leq \alpha_{n}\left(\left\|T^{n} y_{n}-T^{n} x_{n}\right\|+\left\|T^{n} x_{n}-x_{n}\right\|\right) \\
& \leq \alpha_{n}\left(L\left\|y_{n}-x_{n}\right\|+\left\|T^{n} x_{n}-x_{n}\right\|\right) \\
& \leq \alpha_{n}\left(1+\beta_{n} L\right)\left\|T^{n} x_{n}-x_{n}\right\| .
\end{aligned}
$$

Using (2.42), we obtain

$$
\lim _{n \rightarrow \infty}\left\|x_{n+1}-x_{n}\right\|=0
$$

Observe that

$$
\begin{aligned}
\left\|x_{n}-T x_{n}\right\| \leq & \left\|x_{n}-x_{n+1}\right\|+\left\|x_{n+1}-T^{n+1} x_{n+1}\right\|+\left\|T^{n+1} x_{n+1}-T^{n+1} x_{n}\right\| \\
& +\left\|T^{n+1} x_{n}-T x_{n}\right\| \\
\leq & (1+L)\left\|x_{n}-x_{n+1}\right\|+\left\|x_{n+1}-T^{n+1} x_{n+1}\right\|+L\left\|T^{n} x_{n}-x_{n}\right\| .
\end{aligned}
$$


Using (2.42) and (2.44), we have

$$
\lim _{n \rightarrow \infty}\left\|x_{n}-T x_{n}\right\|=0
$$

Since $\left\{x_{n}\right\}$ is bounded, the sequence $\left\{T x_{n}\right\}$ has a convergent subsequence $\left\{T x_{n_{r}}\right\}$ say. Let $T x_{n_{r}} \rightarrow q$ as $r \rightarrow \infty$. Then $x_{n_{r}} \rightarrow q$ as $r \rightarrow \infty$ since

$$
0=\lim _{r \rightarrow \infty}\left[x_{n_{r}}-T x_{n_{r}}\right]=\lim _{r \rightarrow \infty} x_{n_{r}}-\lim _{r \rightarrow \infty} T x_{n_{r}}=\lim _{r \rightarrow \infty} x_{n_{r}}-q
$$

By the continuity of $T, T x_{n_{r}} \rightarrow T q$ as $r \rightarrow \infty$ but $T x_{n_{r}} \rightarrow q$ as $r \rightarrow \infty$. Hence, $q=T q$.

Hence, $\left\{x_{n}\right\}_{n=0}^{\infty}$ has a subsequence which converges to the fixed point $q$ of $T$. Using (2.38), there exists some natural number $N$, when $n>N,\left(1-\beta_{n}-\beta_{n}^{2} L^{2}\right)-\beta_{n} a_{n}^{2} \geq \frac{1-2 b-b^{2} L^{2}}{2}>0$ $\forall n>N$. Using (2.36), $0 \leq \alpha_{n}\left(1+\beta_{n} a_{n}^{2}\right) v_{n}+\alpha_{n}\left(1+\beta_{n} a_{n}^{2}\right)\left(a_{n}^{2}-1\right)\left\|x_{n}-q\right\|^{2} \leq M\left(a_{n}^{2}-1\right)+M v_{n}$. From (2.35),

$$
\left\|x_{n+1}-q\right\|^{2} \leq\left\|x_{n}-q\right\|^{2}+M\left(a_{n}^{2}-1+v_{n}\right)
$$

But $\sum_{n=1}^{\infty}\left(a_{n}^{2}-1\right)<+\infty$ and $\sum_{n=1}^{\infty} v_{n}<+\infty$ imply that $\sum_{n=1}^{\infty} M\left(a_{n}^{2}-1+v_{n}\right)<+\infty$. From (2.47), it follows that there exists a subsequence $\left\{\left\|x_{n_{r}}-q\right\|^{2}\right\}_{r=1}^{\infty}$ of $\left\{\left\|x_{n}-q\right\|^{2}\right\}_{n=1}^{\infty}$, which converges to 0 . Hence, using (2.48) and Lemma $1.2, \lim _{n \rightarrow \infty}\left\|x_{n}-q\right\|^{2}=0$. This means that $\lim _{n \rightarrow \infty} x_{n}=q$. The proof of the theorem is complete.

Observe that if $v_{n}=0$ for all $n \in \mathbb{N}$ in Theorem 2.6, then we obtain Theorem 3 of Qihou [2].

Corollary 2.7 [2, Theorem 3] Let $H$ be a Hilbert space, $C \subset H$ be nonempty closed bounded and convex; $T: C \rightarrow C$ be completely continuous and uniformly L-Lipschitzian and asymptotically hemicontractive with sequence $\left\{a_{n}\right\}, a_{n} \in[1,+\infty) ; \forall n \in \mathbb{N}, \sum_{n=1}^{\infty}\left(a_{n}-\right.$ 1) $<+\infty ;\left\{\alpha_{n}\right\},\left\{\beta_{n}\right\} \in[0,1] ; \epsilon \leq \alpha_{n} \leq \beta_{n} \leq b$ for $\forall n \in \mathbb{N}$, some $\epsilon>0$, and some $b \in$ $\left(0, L^{-2}\left[\left(1+L^{2}\right)^{\frac{1}{2}}-1\right]\right) ; x_{1} \in C$ for $\forall n \in \mathbb{N}$ define

$$
\left\{\begin{array}{l}
z_{n}=\beta_{n} T^{n} x_{n}+\left(1-\beta_{n}\right) x_{n}, \\
x_{n+1}=\alpha_{n} T^{n} z_{n}+\left(1-\alpha_{n}\right) x_{n}, \quad n \geq 1 .
\end{array}\right.
$$

Then $\left\{x_{n}\right\}$ converges strongly to some fixed point of $T$.

Since the class of asymptotically pseudocontractive mappings in the intermediate sense is a subclass of the class of asymptotically hemicontractive mappings in the intermediate sense, we obtain the following corollary.

Corollary 2.8 [1, Theorem 2.1] Let $C$ be a nonempty, closed and convex subset of a real Hilbert space $H$ and $T: C \rightarrow C$ be a uniformly L-Lipschitzian and asymptotically pseudocontractive mapping in the intermediate sense with sequences $\left\{k_{n}\right\} \subset[1, \infty)$ and $\left\{\tau_{n}\right\} \subset[0, \infty)$ as defined in (1.11). Assume that the interior of $F(T)$ is nonempty. Let $\left\{x_{n}\right\}$ be a sequence defined by $x_{1}=x \in C$ and

$$
\left\{\begin{array}{l}
y_{n}=\beta_{n} T^{n} x_{n}+\left(1-\beta_{n}\right) x_{n}, \\
x_{n+1}=\alpha_{n} T^{n} y_{n}+\left(1-\alpha_{n}\right) x_{n}, \quad n \geq 1,
\end{array}\right.
$$


where $\left\{\alpha_{n}\right\}$ and $\left\{\beta_{n}\right\}$ are sequences in $(0,1)$. Assume that the following conditions are satisfied:

(i) $\sum_{n=1}^{\infty} \tau_{n}<\infty, \sum_{n=1}^{\infty}\left(q_{n}^{2}-1\right)<\infty$, where $q_{n}=2 k_{n}-1$;

(ii) $a \leq \alpha_{n} \leq \beta_{n} \leq b$ for some $a>0$ and $b \in\left(0, L^{-2}\left[\sqrt{1+L^{2}}-1\right]\right)$.

Then the sequence $\left\{x_{n}\right\}$ generated by (2.49) converges strongly to a fixed point of $T$.

\section{Competing interests}

The authors declare that they have no competing interests.

\section{Authors' contributions}

All authors contributed equally and significantly in this research work. All authors read and approved the final manuscript.

\section{Acknowledgements}

The authors thank the referees for their painstaking and valuable comments and corrections which lead to the improvement of this manuscript.

Received: 2 October 2012 Accepted: 21 November 2013 Published: 28 December 2013

\section{References}

1. Zegeye, H, Robdera, M, Choudhary, B: Convergence theorems for asymptotically pseudocontractive mappings in the intermediate sense. Comput. Math. Appl. 62, 326-332 (2011)

2. Qihou, $\mathrm{L}$ : Convergence theorems of the sequence of iterates for asymptotically demicontractive and hemicontractive mappings. Nonlinear Anal., Theory Methods Appl. 26(11), 1835-1842 (1996)

3. Sahu, DR, Xu, HK, Yao, J-C: Asymptotically strict pseudocontractive mappings in the intermediate sense. Nonlinear Anal., Theory Methods Appl. 70(10), 3502-3511 (2009)

4. Qin, X, Cho, SY, Kim, JK: Convergence theorems on asymptotically pseudocontractive mappings in the intermediate sense. Fixed Point Theory Appl. 2010, Article ID 186874 (2010). doi:10.1155/2010/186874

5. Schu, J: Iterative construction of fixed points of asymptotically nonexpansive mappings. J. Math. Anal. Appl. 158, 407-413 (1991)

6. Liu, Q: On Naimpally and Singh's open questions. J. Math. Anal. Appl. 124, 157-164 (1987)

7. Hicks, TL, Kubicek, JD: On the Mann iteration process in a Hilbert spaces. J. Math. Anal. Appl. 59, $498-504$ (1977)

8. Cho, YJ, Zhou, HY, Kang, SM: Strong convergence theorems for iterative schemes with errors for asymptotically demicontractive mappings in arbitrary real normed linear spaces. J. Inequal. Pure Appl. Math. 3, 1-11 (2002)

9. Maruster, L, Maruster, S: Strong convergence of the Mann iteration for $\alpha$-demicontractive mappings. Math. Comput. Model. 54, 2486-2492 (2011)

10. Olaleru, JO, Mogbademu, AA: On the modified Noor iteration scheme for non-linear maps. Acta Math. Univ. Comen. $\operatorname{LXXX(2),~221-228~(2011)~}$

11. Olaleru, JO, Mogbademu, AA: Approximation of fixed points of strongly successively pseudocontractive maps in Banach space. Int. J. Comput. Appl. Math. 7(2), 121-132 (2012)

12. Osilike, MO: Iterative approximation of fixed points of asymptotically demicontractive mappings. Indian J. Pure Appl. Math. 24, 1291-1300 (1998)

13. Osilike, MO, Aniagbosor, SC: Fixed points of asymptotically demicontractive mappings in certain Banach spaces. Indian J. Pure Appl. Math. 32, 1519-1537 (2001)

14. Moore, C, Nnoli, BVC: Iterative sequence for asymptotically demicontractive maps in Banach spaces. J. Math. Anal. Appl. 302, 557-562 (2005)

15. Olaleru, JO, Okeke, GA: Strong convergence theorems for asymptotically pseudocontractive mappings in the intermediate sense. Br. J. Math. Comput. Sci. 3(2), 151-162 (2012)

16. Igbokwe, DI: Approximation of fixed points of asymptotically demicontractive mappings in arbitrary Banach spaces. J. Inequal. Pure Appl. Math. 3(1), Article ID 3 (2002)

doi:10.1186/1687-1812-2013-352

Cite this article as: Olaleru and Okeke: Convergence theorems on asymptotically demicontractive and hemicontractive mappings in the intermediate sense. Fixed Point Theory and Applications 2013 2013:352. 